\title{
Entice With Procedures, Inspire With Primary Care: A Preclerkship Pipeline Course
}

Crystal Lin, MS* | Jimmy Zheng, BS* | Vinita Shivakumar, BS* | Erika Schillinger, MD | Tracy A. Rydel, MD | Tamara Montacute, MD, MPH

PRiMER. 2021;5:22.

Published: 6/28/2021 | DOI: 10.22454/PRiMER.2021.782026

\section{Abstract}

Background and Objectives: The growing demand for primary care clinicians in the United States continues to outstrip their dwindling supply. Many allopathic medical schools, including Stanford University School of Medicine, are not adequately meeting this shortage. We sought to develop a preclerkship elective to increase the visibility and desirability of primary care at our institution.

Methods: A novel 9-week preclerkship elective titled "Primary Care Defined: Perspectives and Procedures," was designed as a series of procedural workshops followed by interactive sessions with local primary care clinicians. A total of 36 medical and physician assistant students were enrolled. We administered a questionnaire pre- and postcourse to evaluate the impact of the elective on learner interest and attitudes toward primary care.

Results: Twenty-four enrolled and 10 nonenrolled learners completed the questionnaire both pre- and postcourse. A one-way analysis of covariance controlling for gender, program (medical doctor versus physician assistant), and precourse responses demonstrated that enrollees had a significantly increased interest in primary care compared to nonenrollees after the course $\left(F_{1,32}=9.22, P=.005\right)$. Enrollees also more positively rated their attitudes toward compensation, scope of practice, and job fulfillment than nonenrollees. Both groups had high levels of agreement on statements concerning patient-physician interactions and the importance of primary care to the health care system.

Conclusion: The design and content of this elective offers a framework for other institutions looking to promote the value of primary care specialties, particularly family medicine. Creating opportunities for experiential learning and early student-faculty engagement may encourage preclerkship learners to consider a career in primary care.

\section{Introduction}

Primary care in the United States faces a growing supply-demand mismatch. By 2033, the Association of American Medical Colleges projects a shortage of 21,100 to 55,200 primary care physicians and a fall in the ratio of physicians to physician assistants (PA) from $2: 1$ to $1: 1 .^{1}$ It remains to be seen whether the rising supply 
of PAs will offset demand for primary care. ${ }^{1}$ However, increasing proportions of US MD and PA graduates choose to subspecialize each year. ${ }^{2-4}$ US medical institutions are not increasing the number of students committed to primary care careers, with less than $10 \%$ of graduates over the past decade entering a family medicine residency. ${ }^{5-6}$

Stanford University School of Medicine is a private academic medical center with 90 MD and 25 PA students annually. From 2011 to 2020 , fewer than $5 \%$ of each graduating class on average matched into family medicine residencies. Although more students enter internal medicine $(21 \%)$ or pediatric $(7 \%)$ residencies, most subspecialize. ${ }^{7}$ Current efforts to foster primary care interest include a patient-centered elective course, student interest groups, and faculty mentorship for research. Clerkship students spend a month in a family medicine rotation and may opt for primary care electives. Despite these opportunities, the pool of students interested in primary care remains modest.

To cultivate early primary care interest among preclerkship students, we developed a novel seminar titled "Primary Care Defined: Perspectives and Procedures." The course was split into procedural workshops and interactive sessions highlighting clinician perspectives to promote experiential learning and professional mentorship. 8,9 While similar educational strategies have been successfully applied to emergency medicine, anesthesiology, and surgical fields, none have been reported for primary care specialties. ${ }^{10}$ We hypothesized that early preclinical exposure to hands-on learning and meaningful clinical interactions would improve enrollees' interest and attitudes toward a career in primary care, as measured by a modified validated Family Medicine Attitudes Questionnaire.

\section{Methods}

\section{Course Design}

This elective was a weekly, 1-hour, lunchtime seminar for Stanford preclerkship students. Following an introductory session, there were four case-based procedural workshops and four interactive sessions. Procedures included abscess incision and drainage, joint injection, intrauterine device insertion, and shave biopsy. Workshops included a patient case, a live demonstration, and small-group practice with an MD or PA facilitator. The interactive sessions addressed key topics such as work-life fit, salary, and posttraining trajectories through panel discussions or breakout groups with clinicians. Speakers from family medicine, internal medicine, and pediatrics across different practice settings were invited. Full details on our curriculum and workshop supplies can be found in the STFM Resource Library. ${ }^{11}$

\section{Outcomes Evaluation}

To evaluate changes in enrollees' attitudes toward primary care, we modified the previously-validated 14-item Family Medicine Attitudes Questionnaire, which covers six domains (patient relationships, competence and expertise, lifestyle, research, specialty importance, and shortage). ${ }^{12}$ Our final unvalidated survey contained 11 5 -point Likert scale items from the original instrument, ${ }^{13}$ with an additional question assessing interest in primary care, scaled from 1 to 7 for greater statistical sensitivity. ${ }^{14}$ We administered the survey during the first and last weeks of the academic quarter. A nonenrolled control group was recruited through school mailing lists. No compensation was offered for completing the questionnaire. We programmed the tool using Qualtrics XM software (Provo, UT).

We used one-way analyses of covariance (ANCOVA) to test the null hypothesis that enrollee attitudes toward primary care were equal to those of control participants. We assigned enrollment status as the fixed factor, with gender, program (MD vs PA), and precourse responses as covariates, based on variables with major differences between enrollees and controls. We also performed an ANOVA of change from baseline to confirm our findings 
due to concern for unconsidered selection bias. ${ }^{15}$ We used SPSS software, version 24.0 (IBM Corp, Armonk, NY) for statistical analysis, and set statistical significance at $P<.05$.

The Stanford University Institutional Review Board determined that this study did not meet the definition of human subjects' research.

\section{Results}

Twenty-four enrolled and 10 nonenrolled learners completed the pre- and postcourse surveys. Nine enrollees (38\%) were MDs and 15 (62\%) were PAs, compared to seven (70\%) and three (30\%) in the nonenrollee group, respectively. Gender distribution was comparable between enrollees ( $71 \%$ female) and nonenrollees $(70 \%$ female). Table 1 shows other demographic information. Precourse responses were not significantly different between enrollees and nonenrollees (Table 2).

The one-way ANCOVA assessing postcourse level of interest in a career in primary care (correcting for gender, program, and pre-course ratings as covariates) demonstrated a statistically significant difference $\left(F_{1,32}=9.22\right.$; $P=.005)$ between enrollees $(5.50$ out of $7 ; 95 \% \mathrm{Cl}, 5.16-5.84)$ and nonenrollees $(4.49 ; 95 \% \mathrm{Cl}, 3.94-5.05)$. The one-way ANOVA of change from baseline was similarly significant $\left(F_{1,19}=5.10 ; P=.036\right)$, differing primarily in effect size. Significant differences were found in four of the 11 statements, specifically about income, job complexity, career enjoyment, and procedural involvement (Table 3). Statements regarding patient relationships and the importance of primary care $(6,9,10,11)$ received high levels of agreement from both groups.

Differences were noted for statements about research and knowledge depth $(5,7,8)$, but were not significant.

\section{Discussion}

This novel curriculum received highly positive feedback. Learners favored the small class size, high faculty-tostudent ratio, and diversity of skills learned. Constructive feedback included allotting more time for the procedural walk-through and reorganizing stations for smoother workflow. Learners also stressed the value of hearing honest perspectives from clinicians on important topics like income and job fulfillment. We observed high levels of engagement across all sessions, lending credibility to the course design and content.

After the course, enrollees rated their interest in primary care significantly higher than did nonenrollees. They also scored more positively on statements regarding compensation, scope of practice, and job fulfillment. These differences suggest that this elective effectively defined key features of primary care and addressed common misconceptions. ${ }^{16}$ Enrollees and nonenrollees had high levels of agreement with statements concerning patient-physician interactions and the value of primary care, suggesting baseline appreciation of this information. Topics such as research opportunities and interactions between generalists and specialists may deserve more emphasis in future iterations.

\section{Limitations}

Students likely enrolled in this course based on preexisting interest in primary care. Other selection factors include interest in procedural exposure or elective credit for graduation. Enrollees may have known more about the domains highlighted during the elective or felt obliged to submit higher ratings despite guarantee of anonymity. Additionally, many enrollees were PA students who might differ from medical students in their educational goals. Effects of precourse scores and program type, however, were controlled for in the ANCOVA. We did not control for home town size, which differed between enrollees and nonenrollees. Notably, a higher proportion of controls came from rural backgrounds, which may correlate with a higher interest in primary care specialties. ${ }^{17}$ 
While the original survey was validated as an instrument to assess fourth-year medical students' attitudes toward family medicine and likelihood of matching into the specialty, we administered a modified version to preclinical medical students without revalidation, thus limiting the ability to make definitive conclusions based on the survey results. Long-term outcomes, such as match rates into primary care specialties, could not be evaluated given the recency of this course and its small sample size. We also did not stratify student responses by individual primary care specialty. The predominance of PA enrollees will additionally limit generalizability to other institutions. Future instructors should longitudinally follow a larger sample of past and future students for the analysis of residency match outcomes and possible validation of the modified survey instrument.

\section{Conclusions}

Our novel elective offers a framework for other medical schools to generate early positive messaging around the activities and impact of primary care specialties. Coupling experiential learning with meaningful studentfaculty engagement may address concerns that deter preclerkship learners from pursuing careers in primary care.

\section{Tables and Figures}


Table 1: Survey Respondent Demographics

\begin{tabular}{|c|c|c|}
\hline \multirow{2}{*}{ Characteristic } & \multicolumn{2}{|c|}{ n (\%) or Median (IQR) } \\
\hline & Enrollees, n=24 & Nonenrollees, $n=10$ \\
\hline \multicolumn{3}{|l|}{ Gender } \\
\hline Female & $17(71)$ & $7(70)$ \\
\hline Male & $7(29)$ & $2(20)$ \\
\hline Other & $0(0)$ & $1(10)$ \\
\hline \multicolumn{3}{|l|}{ Program } \\
\hline Medical student & $9(38)$ & $7(70)$ \\
\hline Physician assistant & $15(62)$ & $3(30)$ \\
\hline \multicolumn{3}{|l|}{ Race $^{a}$} \\
\hline White & $13(52)$ & $8(73)$ \\
\hline Black or African American & $1(4)$ & $0(0)$ \\
\hline Asian & $9(36)$ & $2(18)$ \\
\hline American Indian or Alaska Native & $1(4)$ & $1(9)$ \\
\hline Other & $1(4)$ & $0(0)$ \\
\hline \multicolumn{3}{|l|}{ Hometown Size } \\
\hline Small city/suburb & $15(63)$ & $3(30)$ \\
\hline Small town & $5(21)$ & $0(0)$ \\
\hline Large city & $3(13)$ & $2(20)$ \\
\hline Rural & $1(4)$ & $5(50)$ \\
\hline \multicolumn{3}{|l|}{ Marital Status } \\
\hline Single, never married & $20(83)$ & $9(90)$ \\
\hline Married or domestic partnership & $3(13)$ & $1(10)$ \\
\hline Widowed & $1(4)$ & $0(0)$ \\
\hline Anticipated Educational Debt ${ }^{b}$ & $\$ 135,000(\$ 170,000)$ & $\$ 150,000(\$ 97,500)$ \\
\hline
\end{tabular}

a Category totals may not add to the group sizes due to multiselect answers.

b Debt (eg, loans) upon graduation from current program.

Abbreviations: IQR, interquartile range. 
Table 2: Precourse Questionnaire Response Comparison

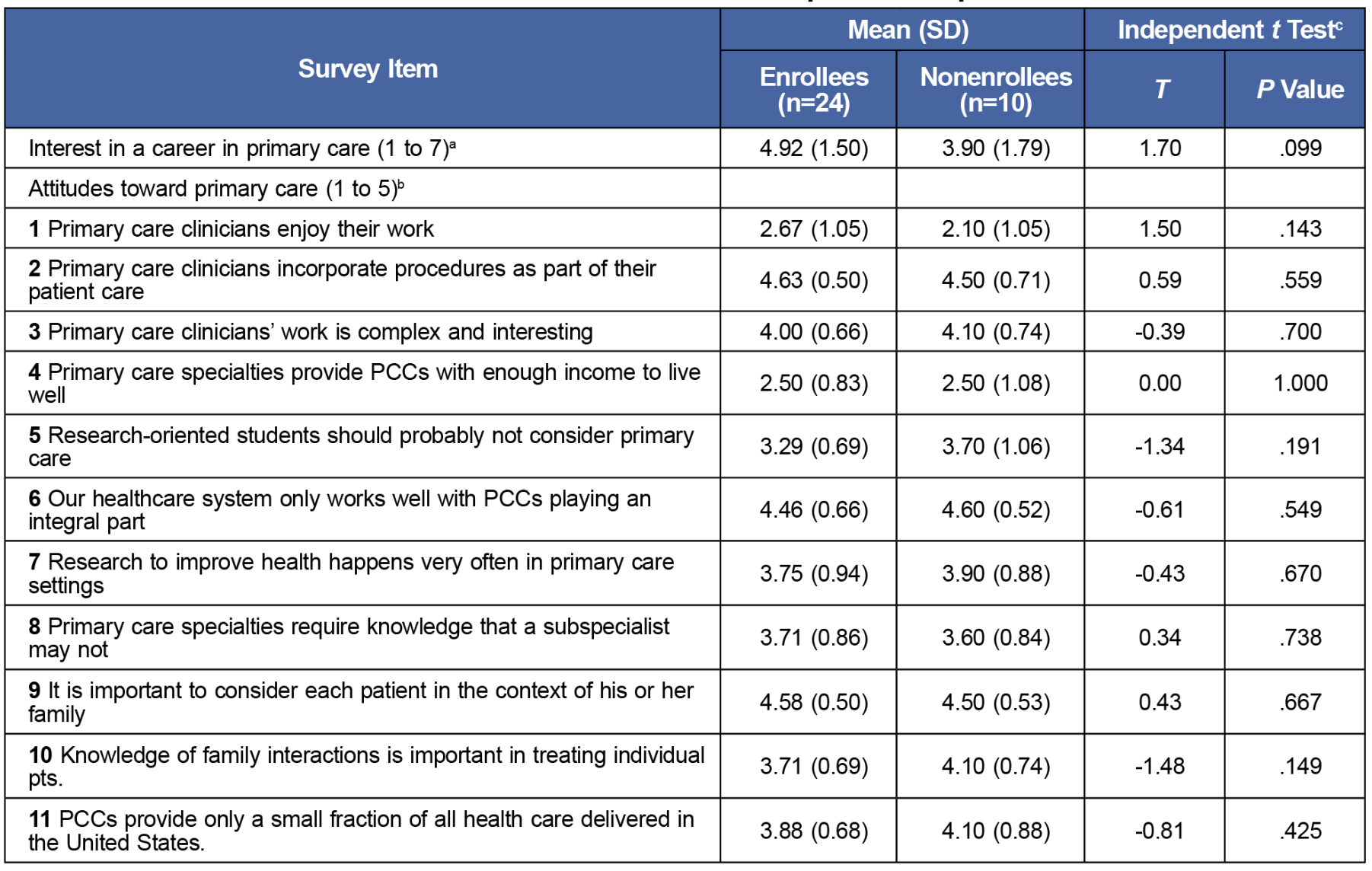

a Interest in primary care career was evaluated on 1 to 7 scale, with 1 lowest and 7 highest

${ }^{b}$ Agreement with survey items was evaluated on a 1 to 5 scale: strongly disagree, disagree, unsure, agree, and strongly agree, respectively.

c Equal variances assumed given significance $>.05$ for all survey items under Levene's Test for Equality of Variances.

Survey items were not presented in the order listed above.

Abbreviations: PCC, primary care clinician. 
Table 3: Postcourse Questionnaire Outcomes

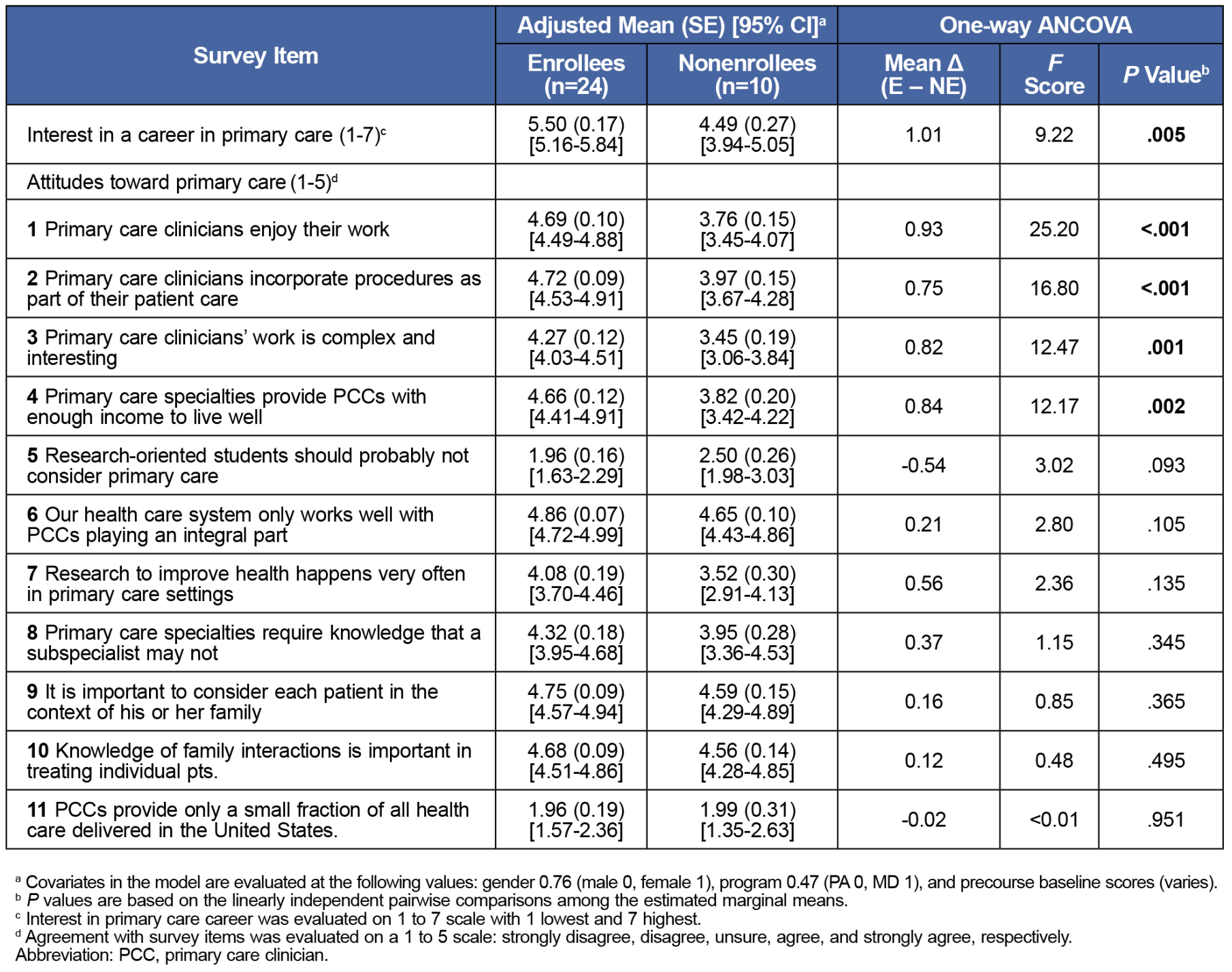

\section{Acknowledgments}

The authors thank the Stanford Division of Primary Care and Population Health administrators, Kim Osborn and Cicily Chirayath, for their continuous logistical support during the execution of this course. They also thank the multiple faculty, residents, and patient partners who graciously volunteered their time to engage learners, as well as Amy Rohman from Bayer HealthCare Pharma for supplying several anatomical models and IUD placebo inserters.

* Authors Crystal Lin, Jimmy Zheng, and Vinita Shivakumar contributed equally to this work.

Presentation: The results of this paper were presented at the Society of Teachers of Family Medicine (STFM) Conference on Medical Student Education, January 31, 2020, in Portland, Oregon.

\section{Corresponding Author}

Tamara Montacute, MD, MPH

Division of Primary Care and Population Health, Department of Medicine, Stanford University School of Medicine, 211 Quarry Rd Ste 405, MC 5985, Palo Alto, CA 94304. 


\section{Author Affiliations}

Crystal Lin, MS* - Stanford University School of Medicine, Stanford, CA

Jimmy Zheng, BS* - Stanford University School of Medicine, Stanford, CA

Vinita Shivakumar, BS* - Stanford University School of Medicine, Stanford, CA

Erika Schillinger, MD - Division of Primary Care and Population Health, Department of Medicine, Stanford University School of Medicine, Palo Alto, CA

Tracy A. Rydel, MD - Division of Primary Care and Population Health, Department of Medicine, Stanford University School of Medicine, Stanford, CA

Tamara Montacute, MD, MPH - Division of Primary Care and Population Health, Department of Medicine, Stanford University School of Medicine, Stanford, CA

\section{References}

1. Association of American Medical Colleges. The complexities of physician supply and demand: projections from 2018 to 2033. Published June 2020. Accessed on March 4, 2021. https://www.aamc.org/system/files/2020-06/stratcomm-aamc-physician-workforce-projectionsjune-2020.pdf

2. National Commission on Certification of Physician Assistants. 2018 Statistical Profile of Certified Physician Assistants. Johns Creek, GA: National Commission on Certification of Physician Assistants; 2019. Accessed March 4, 2021. https://prodcmsstoragesa.blob.core.windows.net/uploads/files /2018StatisticalProfileofCertifiedPhysicianAssistants.pdf

3. Hooker RS, Cawley JF, Everett CM. Predictive modeling the physician assistant supply: 2010-2025. Public Health Rep. 2011;126(5):708-716. doi:10.1177/003335491112600513

4. Newton DA, Grayson MS. Trends in career choice by US medical school graduates. JAMA. 2003;290(9):1179-1182. doi:10.1001/jama.290.9.1179

5. Phillips JP, Wendling A, Bentley A, Marsee R, Morley CP. Trends in US medical school contributions to the family physician workforce: 2018 update from the American academy of family physicians. Fam Med. 2019;51(3):241-250. doi:10.22454/FamMed.2019.395617

6. Messinger CJ, Hafler J, Khan AM, Long T. Recent trends in primary care interest and career choices among medical students at an academic medical institution. Teach Learn Med. 2017;29(1):42-51. doi:10.1080/10401334.2016.1206825

7. Petterson S, McNellis R, Klink K, Meyers D, Bazemore A. The State of Primary Care in the United States: A Chartbook of Facts and Statistics. Published January 2018. Accessed March 13, 2021. https://www.graham-center.org/content/dam/rgc/documents/publications-reports/reports /PrimaryCareChartbook.pdf

8. Tough A, Knowles MS. Andragogy in action: applying modern principles of adult learning. J Higher Educ. 1985;56(6):707. doi:10.2307/1981081

9. Indyk D, Deen D, Fornari A, Santos MT, Lu W-H, Rucker L. The influence of longitudinal mentoring on medical student selection of primary care residencies. BMC Med Educ. 2011;11(1):27. doi:10.1186/1472-6920-11-27

10. Agarwal A, Wong S, Sarfaty S, Devaiah A, Hirsch AE. Elective courses for medical students during the preclinical curriculum: a systematic review and evaluation. Med Educ Online. 2015;20(1):26615. doi:10.3402/meo.v20.26615

11. Lin C, Zheng J, Shivakumar V, Schillinger E, Rydel T, Montacute, TK. "Primary Care Defined: Perspectives and Procedures" Course Curriculum. STFM Resource Library. March 1, 2021. Accessed June 23, 2021. https://resourcelibrary.stfm.org/viewdocument/primary-care-definedperspectives?CommunityKey=2751b51d-483f-45e2-81de-4faced0a290a\&tab=librarydocuments 
12. Phillips JP, Prunuske J, Fitzpatrick L, Mavis B. The family medicine attitudes questionnaire: A valid instrument to assess student attitudes toward family medicine. Fam Med. 2019;51(6):493-501. doi:10.22454/FamMed.2019.957692

13. Phillips J, Prunuske J, Mavis LF. Family Medicine Survey. STFM Resource Library. Decemer 4, 2017. Accessed July 1, 2019. https://resourcelibrary.stfm.org/viewdocument/family-medicine-survey

14. Finstad K. Response interpolation and scale sensitivity: evidence against 5-point scales. J Usability Stud. 2010;5(3):104-110.

15. Van Breukelen GJP. ANCOVA versus change from baseline: more power in randomized studies, more bias in nonrandomized studies [corrected]. J Clin Epidemiol. 2006;59(9):920-925. doi:10.1016/j.jclinepi.2006.02.007

16. Beverly EA, Wietecha DA, Nottingham K, Rush LJ, Law TD. Premedical students' attitudes toward primary care medicine. J Am Osteopath Assoc. 2016;116(5):302-309. doi:10.7556/jaoa.2016.060

17. Senf $\mathrm{JH}$, Campos-Outcalt $\mathrm{D}$, Kutob R. Factors related to the choice of family medicine: a reassessment and literature review. J Am Board Fam Pract. 2003;16(6):502-512. doi:10.3122/jabfm.16.6.502

Copyright $(\subseteq 2021$ by the Society of Teachers of Family Medicine 